\title{
Staatliche Eingriffe in den chinesischen Immobilienmarkt - Fragen der Rechtmäßigkeit und des Rechtsschutzes
}

\author{
Von Björn Ahl, Köln*
}

\section{A. Einleitung}

In über drei Jahrzehnten der Reform- und Öffnungspolitik hat sich China in seiner Wirtschaftspolitik als ein flexibler, adaptiver und zugleich autoritärer Staat erwiesen. Dieser Erfolg geht so weit, dass dieses als „Beijing Consensus“ ${ }^{\text {“1 }}$ bezeichnete Entwicklungsmodell von vielen Entwicklungs- und Schwellenländern als eine attraktive Alternative zur marktwirtschaftlichen Demokratie angesehen wird. ${ }^{2}$ Auch hat China die Einflüsse der globalen Finanz- und Wirtschaftskrise 2008/2009 entgegen negativen Prognosen, die einen starken Wachstumseinbruch mit unberechenbaren sozialen und politischen Folgen vorhersagten, durch aggressive Anti-Krisen-Maßnahmen des Parteistaats abgewehrt. ${ }^{3}$ Die Rechtspositionen der Wirtschaftssubjekte scheinen bei diesen oft einschneidenden Maßnahmen von untergeordneter Bedeutung zu sein.

Im westlichen Schrifttum wird vielfach die Frage erörtert, wie ein über Jahrzehnte konstant hohes Wirtschaftswachstum auf der Grundlage eines Rechtssystem stattfinden kann, in dem der Eigentumsschutz und die Möglichkeiten der Durchsetzung von vertraglichen Vereinbarungen nur schwach ausgeprägt ist. ${ }^{4}$ Diesen Untersuchungen liegt die Hypothese zugrunde, dass eine funktionierende Marktwirtschaft nur unter den Bedingungen des Schutzes von Privateigentum vor staatlichen Enteignungen und der Möglichkeit der gerichtlichen Durchsetzung von vertraglich begründeten Ansprüchen existieren kann. Im Widerspruch zu dieser Annahme scheint der Erfolg des autoritären chinesischen Entwicklungsmodells gerade in einer experimentellen und flexiblen Wirtschaftspolitik begründet zu sein. Diese Wirtschaftspolitik wird nicht vornehmlich durch eine einheitliche nationale Rechtsetzung imple-

* Björn Ahl, Dr. iur. (Heidelberg), Juniorprofessor für chinesische Rechtskultur, Ostasiatisches Seminar, Universität zu Köln, Email: bjoern.ahl@uni-koeln.de. Der Beitrag beruht auf einem Vortrag auf der Tagung „Wettbewerb und staatliche Intervention“ im September 2011 an der National Taiwan University in Taibei. Für wertvolle Hinweise danke ich Xie Libin, Zhao Hong und Daniel Sprick. Bei der Recherche und Fertigstellung des Manuskripts haben Pilar Czoske, Chen Dachuang, Fang Ming und Mira Luthe geholfen.

1 Im Gegensatz zum "Washington Consensus", Joshua Cooper Ramo, The Beijing Consensus, London 2004.

2 Sebastian Heilmann, Die Volksrepublik China als lernendes autoritäres System: Experimentierende Staatstätigkeit und wirtschaftliche Modernisierung, NZZ 28./29. Juni 2008.

3 Siehe Dirk Schmidt, Die Volksrepublik China und die internationale Finanzkrise, China Analysis 75, Oktober 2009, http://chinapolitik.de/resources/no_75.pdf.

4 Donald Clarke, Economic Development and the Rights Hypothesis: The China Problem, American Journal of Comparative Law Vol. 51 (2003), S. 89-111. 
mentiert, sondern oftmals unter Verstoß gegen höherrangiges Recht und durch Eingriffe in die durch nationales Recht begründeten Rechtspositionen der Wirtschaftssubjekte auf lokaler Ebene durchgesetzt.

In der Herrschaftsperiode Mao Zedongs stützte sich die Verwaltungstätigkeit in der Volksrepublik allein auf so genannte ,rotköpfige Dokumente“ ${ }^{5}$ die Zielvorgaben der parteistaatlichen Führung für die unteren Verwaltungsebenen enthielten. Die lokalen Verwaltungsbehörden hatten bei der Umsetzung dieser Ziele ein weites Ermessen. Nach der Kulturrevolution erfolgte eine Abwendung vom dezisionistischen Verwaltungsstil Maos hin zu einer rationalen und an Gesetze gebundenen Verwaltung. ${ }^{6}$ Die Hinwendung des chinesischen Staates zu mehr Rechtssicherheit und Vorhersehbarkeit des Verwaltungshandelns wurde im Jahr 1999 durch die Einführung des Konzepts des „sozialistischen Rechtsstaats“ in die Verfassung dokumentiert. ${ }^{7}$ Trotz des tiefgreifenden Wandels von Wirtschaft und Gesellschaft hat sich die Grundstruktur der Ein-Partei-Herrschaft nicht geändert. Sie kommt vor allem darin zum Ausdruck, dass Regierungsorgane grundsätzlich in ihren Entscheidungskompetenzen Parteikomitees untergeordnet sind, alle wichtigen Führungspositionen im Staat von der Kommunistischen Partei besetzt und Wahlen manipuliert werden. ${ }^{8}$ Vor diesem Hintergrund stellt sich die Frage, wie es mit den Anforderungen an eine gesetzmäßige Verwaltung vereinbart wird, dass Wirtschaftspolitik im Rahmen des chinesischen Ein-Partei-Systems eine sehr flexible Durchsetzung verlangt.

Im Folgenden soll anhand eines behördlichen Eingriffs in den Immobilienmarkt aufgezeigt werden, welche rechtlichen Probleme die Umsetzung solcher interventionistischer wirtschaftspolitischer Entscheidungen in China aufwirft. Es werden dabei Maßnahmen der Zentralregierung und deren Umsetzung in der regierungsunmittelbaren Stadt Peking ${ }^{9}$ untersucht, welche den Erwerb von Eigentumswohnungen durch Private beschränken. Diese Maßnahmen dienen im Bündel mit einer Reihe von weiteren Schritten dazu, die Nachfrage auf dem Immobilienmarkt zu reduzieren und damit die Preise für Wohnimmobilien in chinesischen Großstädten zu stabilisieren.

5 Hongtou wenjian (Rotköpfige Dokumente). Vgl. Robert Heuser, Das chinesische Verwaltungswiderspruchsgesetz von 29.4.1999: Gegen die Willkür der „rotköpfigen Dokumente“, China aktuell (2000) Nr. 2, S. 155-172.

6 Yifa xingzheng (Verwaltung auf der Grundlage von Gesetzen). Vgl. Robert Heuser, „Sozialistischer Rechtsstaat" und Verwaltungsrecht in der Volksrepublik China, Hamburg 2003, S. 24.

7 Vgl. dazu Randall Peerenboom, China's Long March toward Rule of Law, Cambridge 2002; Heuser, Fn. 6, S. 24.

8 Sebastian Heilmann, Das Politische System der Volksrepublik China, Wiesbaden 2004, S. 90.

9 Peking, Shanghai, Tianjin und Chongqing werden als regierungsunmittelbare Städte (zhixiashi) bezeichnet, da sie auf Provinzebene angesiedelt sind und direkt der Zentralregierung unterstehen. 


\section{B. Maßnahmen zur Beschränkung des Erwerbs von Wohnimmobilien}

In den letzten zehn Jahren sind die Preise für Immobilien in China sehr schnell angestiegen, vor allem in den großen Städten im Osten des Landes. Hinsichtlich der Immobilienmärkte in Peking, Shanghai, Shenzhen, Hangzhou und Ningbo wird von einer Immobilienblase gesprochen, die Maßnahmen der Preissteuerung erforderlich machen. ${ }^{10}$ Bereits 2006 hatten einige Abteilungen des Staatsrats gemeinsam ein Rundschreiben erlassen, welches Investitionen von nicht am Ort ansässigen Unternehmen und Einzelpersonen in den Wohnungsmarkt beschränkte. ${ }^{11}$ Diese Beschränkung wurde als eine Reaktion auf die Finanz- und Wirtschaftskrise in einigen Städten Anfang 2009 wieder aufgehoben. ${ }^{12}$ Die Preissteigerung von Immobilien hatte im April 2010 ihren vorläufigen Höhepunkt von 12,8 Prozent im Vergleich zum Vorjahr erreicht. ${ }^{13}$ Durchschnittliche Quadratmeterpreise für neue Eigentumswohnungen in Peking betrugen 2011 18.000 RMB. ${ }^{14}$ Mit einem durchschnittlichen Jahreseinkommen in Peking von 32.903 RMB im Jahr 2011 sind Eigentumswohnungen für den Durchschnittsverdiener nicht erschwinglich. ${ }^{15}$

10 Huayi Yu, Size and Characteristics of Housing Bubbles in China's Major Cities: 1999-2000, China \& World Economy Vol. 19 (2011) Nr. 6, S. 56-75.

11 Jianshebu, shangwubu, fazhan gaigewei, zhongguo renmin yinhang, zhongguo gongshang guanli zongju, guojia waihui guanliju deng liu buwei guanyu guifan fangdichan shichang waizi zhuru he guanli de yijian (Ansichten des Bauministeriums, des Handelsministeriums, der Kommission für Entwicklung und Reform, der chinesischen Volksbank, des Staatsamtes für Industrie und Handel und des Staatsamtes für Devisenverwaltung über die Regulierung des Zugangs und der Verwaltung ausländischer Investitionen im Immobilienmarkt) vom 11.7.2006.

12 Für Peking vgl. Beijingshi guanyu guanche banfa (2008) 131 hao wenjian jingshen zujin benshi fangdichan shichang jiankang fazhan de shishi yijian (Ausführungsansichten der Stadt Peking zur Durchführung des Geistes des Dokuments Nr. 131 (2008) der Staatsratskanzlei über die Förderung der gesunden Entwicklung des Immobilienmarktes) vom 23.1.2009.

13 Die Preise in 70 Großstädten stiegen im Dezember 2010 durchschnittlich um 6,4 Prozent gegenüber dem Vorjahr. Im November 2010 sind sie noch um 7,7 Prozent gestiegen. Vgl. Hu Yuanyuan, Beijing Tightens Restrictions on Flat Buying, China Daily vom 17.2.2011, http://www.chinadaily.com.cn/ business/2011-02/17/content_12030234.htm. Laut der offiziellen Statistik sind die Preise im Jahr 2011 wieder gesunken, 2011 nian guomin jingji he shehui fazhan tongji gongbao (Amtsblatt der Statistik über volkswirtschaftliche und gesellschaftliche Entwicklung im Jahr 2011), http:// www.stats.gov.cn/tjgb/ndtjgb/qgndtjgb/t20120222_402786440.htm.

14 Nach Angaben des Vereins für den Immobilienmarkt Peking betrug der Durchschnittspreis für neu gebaute Eigentumswohnungen (ohne Sozialwohnungen) in der ersten Jahreshälfte 2011 18.297 RMB pro Quadratmeter, http://finance.people.com.cn/GB/15160871.html.

15 Beijing tongji xinxiwang (Statistisches Informationsnetz der Stadt Peking), http://www.bjstats.gov.cn/tjzn/mcjs/201103/t20110314_197736.htm. 
Um die Wohnungspreise zu stabilisieren, hat die Kanzlei des Staatsrats seit 2010 verschiedene Rundschreiben zur Steuerung des Wohnungsmarktes erlassen, ${ }^{16}$ die von den Regierungen der Großstädte umgesetzt wurden. Das Rundschreiben des Staatsrats vom 26.1.2011 mit der Bezeichnung „Mitteilung der Kanzlei des Staatsrats über Fragen betreffend der Kontrolle des Wohnungsmarktes" wendet sich an die dem Staatsrat unmittelbar untergeordneten Staatsorgane, wie auch die Regierung der Stadt Peking. Das Rundschreiben sieht ein Bündel von Maßnahmen vor, um „die Wohnungsprobleme der Stadtbewohner zu lösen“ und die „stabile und gesunde Entwicklung des Immobilienmarktes zu fördern.“ Die Staatsorgane auf Provinzebene werden aufgefordert, den sozialen Wohnungsbau zu fördern und im Jahr 2011 zehn Millionen Sozialwohnungen bereitzustellen. Die Steuerbehörden werden zum Vollzug der Besteuerung von Immobilienveräußerungen aufgefordert. Das Rundschreiben legt auch fest, dass die Konditionen für die Vergabe von Immobilienkrediten danach zu differenzieren sind, ob es sich um einen Kredit für den ersten oder den zweiten Immobilienerwerb handelt. Für Haushalte, die einen Immobilienkredit für eine zweite Wohnimmobilie aufnehmen möchten, beträgt der Eigenkapitalanteil mindestens 60\%; der Zinssatz für Immobilienkredite wird auf mindestens das 1,1-fache des Basiszinssatzes festgelegt. ${ }^{17}$ Ferner macht das Rundschreiben Vorgaben für ein abgestuftes Erwerbsverbot von Immobilien, das an den Ort der Haushaltsregistrierung und die Anzahl von Wohnungen pro Haushalt anknüpft. Bestimmte Städte, die noch keine Maßnahmen zur Beschränkung des Immobilienerwerbs getroffen haben, werden in dem Rundschreiben verpflichtet, entsprechende Maßnahmen innerhalb von drei Wochen zu erlassen.

Um dieser Aufforderung nachzukommen, erließ die Kanzlei der Pekinger Stadtregierung am 15.2.2011 ein ebenfalls als „Mitteilung“ bezeichnetes Rundschreiben, das ein abgestuftes Erwerbsverbot für Wohnimmobilien im Sinne der Vorgaben des Staatsrats enthält. ${ }^{18}$ Dabei stellt die Regelung zwar auf Haushalte als Bezugsgröße ab, die Immobilien werden aber von Einzelpersonen erworben; die Erwerbsbeschränkungen erstrecken sich also auf alle Mitglieder des Haushalts. Demnach ist der Erwerb einer Wohnimmobilie nur solchen Haushalten erlaubt, die in Peking registriert und bislang Eigentümer von nicht mehr als einer Immobilie sind. Haushalte, die lediglich vorübergehend in Peking registriert sind, noch nicht Wohnungseigentümer sind und nachweisen können, dass sie für mindestens fünf Jahre in Peking

16 Guowuyuan bangongting guanyu jinyibu zuohao fangdichan shichang tiaokong gongzuo youguan wenti de tongzhi (Mitteilung der Kanzlei des Staatsrats über Fragen betreffend die Kontrolle des Wohnungsmarktes) vom 26.1.2011; davor hatte der Staatsrat bereits die folgende Anordnung erlassen: Guowuyuan guanyu jianjue ezhi bufen chengshi fangjia guokuai shangzhuang de tongzhi (Mitteilung des Staatsrats über die strikte Kontrolle des zu schnell ansteigenden Wohnungspreises in einem Teil von Städten) vom 17.4.2010.

17 Ziff. 2 bis 4 des Rundschreibens.

18 Vgl. Ziff. 6 der Beijingshi renmin zhengfu bangongting guanyu guanche luoshi guowuyuan bangongting wenjian jingshen jinyibu jiaqiang benshi fangdichan shichang tiaokong gongzuo de tongzhi (Mitteilung der Kanzlei der Volksregierung der Stadt Peking über die Implementierung des Geistes der Dokumente der Kanzlei des Staatsrats zur allmählichen Verstärkung der Kontrolle des Wohnungsmarktes in Peking) vom 15.2.2011. 
Einkommenssteuern oder Sozialversicherungsbeiträge bezahlt haben, können ebenfalls eine Wohnimmobilie erwerben. Der Erwerb von Wohnimmobilien wird allen Haushalten untersagt, die in Peking registriert und Eigentümer von zwei oder mehr Wohnimmobilien sind. Ferner allen Haushalten, die nur vorübergehend in Peking registriert und bereits Eigentümer einer Wohnimmobilie sind oder noch keine Eigentumswohnung haben, jedoch den Nachweis über die Zahlung von Einkommenssteuern oder Sozialversicherungsbeiträgen nicht erbringen können.

Hinsichtlich des Immobilienerwerbs durch Ausländer hatte der Staatsrat schon im November 2010 verfügt, dass Einzelpersonen mit ausländischer Staatsangehörigkeit der Erwerb von nur einer Eigentumswohnung zu Wohnzwecken erlaubt ist. Tochterunternehmen oder Repräsentanzen ausländischer Unternehmen ist in China nur der Kauf von Büroräumen am Ort ihrer Registrierung erlaubt. ${ }^{19}$

\section{Rechtmäßigkeit des Erwerbsverbots}

\section{Form des Verwaltungshandelns}

Für die Beurteilung der Rechtmäßigkeit des Rundschreibens der Stadtregierung ist die Handlungsform bedeutsam, derer sich die Verwaltung hier bedient hat. Das Rundschreiben der Pekinger Stadtregierung wendet sich ausdrücklich an die untergeordneten Behörden und ist als „Mitteilung“ bezeichnet. Der Form nach kommt deshalb eine Qualifizierung als Innenrecht in Betracht, das nur für die angesprochenen Behörden verbindlich ist und keine Außenwirkung entfaltet. Jedoch ist der Tatbestand des Verbots des Immobilienerwerbs für bestimmte Personengruppen auf Außenwirkung gerichtet. In der Praxis ziehen die Gerichte diesen Tatbestand des Rundschreibens auch als Entscheidungsgrundlage heran. ${ }^{20}$

Der Erlass abstrakt-genereller Regelungen durch die Verwaltung wird als administrative Rechtsetzung bezeichnet, wenn es sich um Verwaltungsrechtsbestimmungen ${ }^{21}$ oder Verwaltungsvorschriften ${ }^{22}$ handelt. In anderen Fällen werden abstrakt-generelle Regelungen ,sonstige normative Dokumente“ genannt. ${ }^{23}$ Bei dem Rundschreiben der Stadtregierung könnte es

19 Guojia waihui guanliju guanyu jinyibu guifan jingwai jigou he geren goufang de tongzhi (Mitteilung des staatlichen Verwaltungsamts für Devisen über die schrittweise Normierung des Immobilienerwerbs durch ausländische Organisationen und Einzelpersonen), 4.11.2010, http://www.safe.gov.cn/ model_safe/laws/law_detail.jsp?ID=80401000000000000,34\&id=4.

202011 Beijing faxue fangdichanfa yanjiu zhongxin chunji luntan zuweihui (Organisationskomitee des Frühlingsforums 2011 des Forschungszentrums für Immobilienrecht an der Peking-Universität), Falü shijiao xia de xiangouling jiexi (Analyse der Anordnung der Erwerbsbeschränkung aus rechtlicher Perspektive), 11; http:/crel.law.pku.edu.cn/filedb/20046303708541/20114275056988.pdf.

21 Verwaltungsrechtsbestimmungen (xingzheng fagui) werden vom Staatsrat erlassen.

22 Verwaltungsvorschriften (guizhang) stellen Außenrecht dar und werden von den Abteilungen des Staatsrats (bumen guizhang) oder den lokalen Regierungen (difang zhengfu guizhang) erlassen.

23 Wegen der in roter Farbe abgefassten Überschrift der sonstigen normativen Dokumente (qita guifanxing wenjian) der Verwaltung auch als ,,rotköpfige Dokumente“ bezeichnet. Vgl. Heuser, Fn. 6. 
sich um lokale Verwaltungsvorschriften oder um ein normatives Dokument handeln. Volksregierungen regierungsunmittelbarer Städte können auf der Grundlage von Gesetzen, Verwaltungsrechtsbestimmungen und lokalen Rechtsbestimmungen ${ }^{24}$ Verwaltungsvorschriften erlassen. ${ }^{25}$ Nach dem Gesetzgebungsgesetz und der Methode der Stadt Peking über den Erlass von Verwaltungsvorschriften ${ }^{26}$ handelt es sich bei dem Rundschreiben nicht um eine Verwaltungsvorschrift, da nach $\S 76$ Abs. 2 des Gesetzgebungsgesetzes und $\S 31$ der Methode zum Erlass von Verwaltungsvorschriften erforderlich ist, dass Verwaltungsvorschriften der Lokalregierungen durch ein vom Bürgermeister unterschriebenes Dekret verkündet werden. Das Rundschreiben wurde jedoch unmittelbar von der Stadtregierung und nicht durch ein entsprechendes Dekret verkündet. Die Mitteilung erfüllt somit nicht die Voraussetzung für den Erlass von Verwaltungsvorschriften. Die Mitteilung entspricht auch nicht den weiteren Verfahrensvoraussetzungen.

Nach $\S 15$ der Bestimmungen über das Verfahren zum Erlass von Verwaltungsvorschriften $^{27}$ muss der Entwurf der Verwaltungsvorschriften bei einer direkten Berührung wichtiger Interessen von Bürgern veröffentlicht werden und entweder Ansichten der Öffentlichkeit eingeholt oder eine Anhörungsversammlung durchgeführt werden. Der Entwurf der Mitteilung wurde weder veröffentlicht noch wurden entsprechende Ansichten eingeholt.

Auch hätten Verwaltungsvorschriften in Paragraphen durchnummeriert sein müssen, was bei der Mitteilung ebenfalls nicht der Fall ist. Ferner werden Verwaltungsvorschriften grundsätzlich als „Bestimmungen“ oder „Methode“ und nicht als „Mitteilung“ bezeichnet. Mangels Erfüllung der Verfahrensvoraussetzungen für den Erlass von Verwaltungsvorschriften liegt kein Fall von administrativer Rechtsetzung im engeren Sinne vor. Vielmehr handelt es sich bei dem Rundschreiben lediglich um ein sonstiges normatives Dokument.

\section{Rechtsgrundlage des Erwerbsverbots}

1. Originäre und abgeleitete exekutive Rechtsetzung

Die Regierung der Stadt Peking ist zuständig für den Erlass normativer Dokumente. Fraglich ist, ob in diesem Fall eine ausreichende Rechtsgrundlage vorhanden war. Nach Art 107 Abs. 1 der Verfassung verkünden die lokalen Volksregierungen von der Kreisebene aufwärts „Entscheidungen und Anordnungen“. Nach $\S 59$ des Organisationsgesetzes für die lokalen Volkskongresse und Volksregierungen ${ }^{28}$ können die Volksregierungen von der Kreisebene

24 Lokale Rechtsbestimmungen (difangxing fagui) werden von den lokalen Volkskongressen erlassen.

$25 \S 73$ Zhonghua renmin gongheguo lifafa (Gesetzgebungsgesetz der VR China) vom 15.3.2000.

26 Beijingshi renmin zhengfu guizhang zhiding banfa (Methode über den Erlass von Verwaltungsvorschriften der Volksregierung der Stadt Peking) vom 29.4.2002.

27 Guizhang zhiding chengxu tiaoli (Bestimmungen über das Verfahren zum Erlass von Verwaltungsvorschriften) vom 16.11.2001.

28 Difang geji renmin daibiao dahui he difang geji renmin zhengfu zuzhifa (Gesetz über die Organisation der lokalen Volkskongresse und Volksregierungen) vom 27.10.2004. 
aufwärts zur Ausführung von Anordnungen übergeordneter staatlicher Verwaltungsorgane Anordnungen erlassen. In China wird zwischen einer originären ${ }^{29}$ und einer abgeleiteten Rechtsetzungsbefugnis ${ }^{30}$ der Exekutive unterschieden. Originäre Rechtsetzung bedeutet, dass ein Verwaltungsorgan aufgrund einer allgemeinen Rechtsetzungskompetenz in der Verfassung und dem einschlägigen Organisationsgesetz Recht setzt. Unter einer abgeleiteten Rechtsetzungsbefugnis wird verstanden, dass ein Verwaltungsorgan aufgrund einer in einem Gesetz bestimmten Befugnis oder aufgrund einer besonderen Ermächtigung eines Volkskongresses rechtsetzend tätig wird. ${ }^{31}$

Da das Regierungssystem von der Exekutive dominiert wird, spielt die originäre Rechtsetzung in der Praxis nach wie vor die weitaus wichtigere Rolle. Mit dem Inkrafttreten des Gesetzgebungsgesetzes $^{32}$ im Jahr 2000 ist die Abgrenzung zwischen abgeleiteter und originärer exekutiver Normsetzung etwas klarer geworden. In der Literatur finden sich auch immer mehr Stimmen, welche die originäre Rechtsetzungsbefugnis einschränkend auslegen. ${ }^{33}$ Eine Ansicht möchte die originäre Rechtsetzung auf die Bereiche der Durchführung von höherrangigem Recht, ${ }^{34}$ dem Erlass von Vorschriften zur Organisation und Verfahren sowie die Leistungsverwaltung beschränken. ${ }^{35}$ Folgt man dieser Ansicht, so kann die Pekinger Stadtregierung allein aufgrund von Art. 107 Abs. 1 der Verfassung und von $§ 59$ des Organisationsgesetzes für die lokalen Volkskongresse und Volksregierungen keine normativen Dokumente im Bereich der Eingriffsverwaltung erlassen. Im Schrifttum wird dies ferner damit begründet, dass andernfalls Verwaltungsorgane sich selbst zum Eingriff in Grundrechte ermächtigen könnten und die Gesetzgebungskompetenz der Volkskongresse vollständig ausgehöhlt würde. Es bedürfe einer konkreten gesetzlichen Ermächtigung des Ständigen Ausschusses des Nationalen Volkskongresses oder einer Ermächtigung des Staatsrates durch Verwaltungsrechtsbestimmung, die festlegt, in welchem Umfang ein normatives Dokument in die Rechte und Interessen von Bürgern eingreifen darf. ${ }^{36}$

29 Zhiquan lifa.

30 Shouquan lifa.

31 Yu Shaoru, Lun wo guo xingzheng lifa zhong de zhiquan lifa (Über originäre Rechtsetzung als Teil administrativer Rechtsetzung in China), Wuhan daxue xuebao zhexue shehui kexueban Vol. 62 (2009) Nr. 1, S. 33-36, 33.

32 Zhonghua renmin gongheguo lifafa (Gesetzgebungsgesetz der VR China) vom 15.3.2000.

33 Vgl. Liu Shen, Yifa xingzheng yu xingzheng lifa (Gesetzmäßige Verwaltung und administrative Rechtsetzung), Zhongguo faxue (2000) Nr. 2, S. 86-94.

34 Exekutive Normsetzung zur Durchführung höherrangigen Rechts sieht das Gesetzgebungsgesetz in $\S \S 56$ Abs. 2 Ziff.1, 71 Abs. 2 und 73 Abs. 2 Ziff.1 vor.

35 Yu Shaoru, Fn. 31, S. 34-35.

36 In einem vergleichbaren Fall des Eingriffs in Rechtspositionen Privater auf der Grundlage sonstiger normativer Dokumente siehe: Yang Shilin, Shili 18: Auyunhui qian hou Beijing jidongche xianxing (Fall 18: Fahrbeschränkungen für Kraftfahrzeuge in Peking vor und nach den Olympischen Spielen) in: Han Dayuan, Zhongguo xianfa shili yanjiu (si) (Studien zu Fällen des chinesischen Verfassungsrechts Bd. 4), Peking 2010, S. 228-234, 230. 
2. Gesetzlich bestimmter Vorbehalt einer Regelung durch Verwaltungsrechtsbestimmungen des Staatsrats oder durch Gesetz

Es gibt in China keinen allgemeinen Gesetzesvorbehalt, der Grundrechtseingriffe nur auf der Grundlage von formellen Gesetzen zulässt. Das Gesetzgebungsgesetz und einfache Gesetze legen aber für bestimmte Sachbereiche fest, dass Regelungen nur durch Verwaltungsrechtsbestimmungen des Staatsrats oder durch ein Gesetz des Ständigen Ausschusses des Nationalen Volkskongresses bzw. ein grundlegendes Gesetz ${ }^{37}$ des Nationalen Volkskongresses getroffen werden können.

Hier liegt zwar ein abstrakt-generelles Verwaltungshandeln der Kanzlei des Staatsrats in Form eines Rundschreibens vor, doch ist darin keine Verwaltungsrechtsbestimmung des Staatsrats zu sehen, da das Rundschreiben nicht entsprechend den formellen Voraussetzungen erlassen wurde. ${ }^{38}$ Auch ist fraglich, ob eine Verwaltungsrechtsbestimmung des Staatsrats in diesem Fall als Ermächtigungsgrundlage ausreichen würde. Sieht man die Erwerbsbeschränkung als eine Einschränkung der im Vertragsgesetz gewährleisteten Vertragsfreiheit an, die das „grundlegende zivilrechtliche System“ betrifft, so wäre eine solche Änderung der Rechtslage nach $\S 8$ Ziff. 7 des Gesetzgebungsgesetzes ${ }^{39}$ nur durch ein Gesetz des Nationalen Volkskongresses oder seines Ständigen Ausschusses zulässig. ${ }^{40}$ Wird die Anwendbarkeit von $\S 8$ des Gesetzgebungsgesetzes verneint, so ergibt sich aber aus anderen Vorschriften der Vorbehalt einer Regelung durch Verwaltungsrechtsbestimmungen des Staatsrats.

Nach dem Vertragsgesetz kann die Vertragsfreiheit nur aufgrund von Verwaltungsrechtsbestimmungen des Staatsrats oder durch ein Gesetz beschränkt werden. ${ }^{41}$ Ein Eingriff in die Vertragsfreiheit ließe sich hier mit der Argumentation ausschließen, dass die Erwerbsbeschränkung lediglich die Eintragung der Rechtsänderung bei der Registerbehörde verbiete. Dies hätte auf den Vertragsabschluss und die Wirksamkeit des Vertrages keinen Einfluss und dem Wohnungskäufer bleibe es unbenommen, die Eintragung zu beantragen und bei Verweigerung der Eintragung vor Gericht gegen die Registerbehörde auf Eintragung zu klagen. In der Literatur wendet man sich aus zwei Gründen gegen dieses formale Argument: Einerseits enttäusche das Erwerbsverbot die Erwartungen der Parteien in die Rechtswirkungen des Immobilienkaufvertrages; andererseits führe das Erwerbsverbot in der Praxis dazu, dass die

37 Jiben falü.

38 Vgl. Xingzheng fagui zhiding chengxu tiaoli (Bestimmungen über das Verfahren beim Erlass von Verwaltungsrechtsbestimmungen) vom 16.11.2001.

$39 \S 8$ Ziff. 7 Zhonghua renmin gongheguo lifafa (Gesetzgebungsgesetz der VR China) vom 15.3.2000 bestimmt, dass ,grundlegende zivilrechtliche Systeme“ nur durch Gesetz geregelt werden dürfen.

40 Liu Liantai, Ping wo guo lifafa diba tiao, dijiu tiao guanyu falü baoliu zhidu (Über das System des Gesetzesvorbehalts in $\S 8$ und 9 des chinesischen Gesetzgebungsgesetztes), Henanshen zhengfa guanli ganbu xueyuan xuebao (2003) Nr. 3, S. 104; Organisationskomitee, Fn. 20, S. 15.

41 Nach $\S 4$ des Zhonghua renmin gongheguo hetongfa (Vertragsgesetz der VR China) vom 15.3.1999 sind die Parteien berechtigt, gemäß dem Recht aus eigenem Willen, Verträge zu schließen. § 7 des Vertragsgesetzes bestimmt, dass die Parteien sich beim Abschluss und der Erfüllung von Verträgen nach Gesetzen und Verwaltungsrechtsbestimmungen richten müssen. 
Verkäufer von Immobilien nur mit solchen Kaufinteressenten einen Vertrag abschließen, die nicht unter das Erwerbsverbot fallen. ${ }^{42}$ Demnach steht das Vertragsgesetz einer Regelung des Erwerbsverbots durch ein sonstiges normatives Dokument entgegen. Gleiches ergibt sich aus $\S 10$ Abs. 2 des Sachenrechtsgesetzes. ${ }^{43}$ Danach können Regelungen des Eintragungsverfahrens für unbewegliche Sachen nur aufgrund von Verwaltungsrechtsbestimmungen des Staatsrats oder eines Gesetzes ergehen. Ein Verbot der Eintragung von Immobilien aufgrund eines sonstigen normativen Dokuments ist rechtswidrig. ${ }^{44}$

In der Literatur wird als mögliche gesetzliche Grundlage für die Erwerbsbeschränkung $\S 30$ des Preisgesetzes diskutiert. Diese Vorschrift ermächtigt die Volksregierungen auf Provinzebene bei stark ansteigenden Preisen wichtiger Produkte preisbeschränkende Maßnahmen zu ergreifen. ${ }^{45}$ Obwohl das Ziel der in Rede stehenden Maßnahmen die Stabilisierung von Immobilienpreisen ist, wird argumentiert, dass die Maßnahmen nicht durch das Preisgesetz gedeckt seien. Die Maßnahmen seien keine unmittelbare Beschränkung von Immobilienpreisen, sondern beschränken Transaktionen von Immobilien, was möglicherweise zur Folge hat, dass Immobilienpreise weniger schnell steigen. ${ }^{46}$

\section{Verfassungswidrigkeit des Erwerbsverbots}

\section{Justiziabilität der Verfassung}

Das Argument der Verfassungswidrigkeit der Erwerbsbeschränkung wurde in der öffentlichen und fachlichen Diskussion immer wieder bemüht. Dem ist aber entgegenzuhalten, dass die Vorschriften der chinesischen Verfassung nicht justiziabel sind. Das ergibt sich etwa aus einer

42 Fu Qilin / Wang Liang, Xiangouling falü wenti tanjiu (Untersuchung des Rechtsproblems der Erwerbsbeschränkung), Zhongguo fangdichan (2011) Nr. 8, S. 65-69, 66.

43 Zhonghua renmin gongheguo wuquanfa (Sachenrechtsgestez der VR China) vom 16.3.2007. Deutsche Übersetzung in Zeitschrift für Chinesisches Recht, Vol. 14 (2007), S. 78-117.

44 Guo Shanshan, Qianxi falü shijiao xia de ,xiangouling“(Einfache Untersuchung des Erwerbsverbots aus rechtlicher Perspektive), Dangdai jingji (2011) Nr. 2, S. 36-37, 37; Fu / Wang, Fn. 42, S. 66.

45 Zhonghua renmin gongheguo jiagefa (Preisgesetz der VR China) vom 29.12.1997. §30 Abs. 1: „Wenn die Preise wichtiger Waren oder Dienstleistungen deutlich steigen oder deutlich steigen könnten, können der Staatsrat und die Volksregierungen der Provinzen, autonomen Gebiete und der regierungsunmittelbaren Städte für einen Teil der Preise die Preisdifferenzen oder die Gewinnsätze begrenzen oder Eingriffe wie eine Meldepflicht für Preiserhöhungen oder die Meldung von Preiskorrekturen zu den Akten durchführen.“ $§ 31$ sieht vor: „Wenn das Gesamtniveau der Marktpreise heftig schwankt oder andere ungewöhnliche Umstände auftreten, kann der Staatsrat landesweit oder für Teilgebiete vorübergehend als Dringlichkeitsmaßnahmen die Befugnis zur Festsetzung von Preisen zentralisieren und Preise teilweise oder ganz einfrieren.".

46 Zheng Shaohua, Xiangouling de falü jieshi (Rechtliche Interpretation der Erwerbsbeschränkung), Faxue (2011) Nr. 4, S. 41-43, 42; Fu /Wang, Fn. 42, S. 66-67. 
Justizauslegung ${ }^{47}$ aus dem Jahr 2009, die Richtern verbindlich vorschreibt, welche Rechtsquellen sie in Urteilen zitieren dürfen. ${ }^{48}$ Es entspricht auch der hergebrachten, aus der Sowjetunion übernommenen Doktrin, dass Verfassungsnormen nicht unmittelbar von Gerichten angewandt werden können. ${ }^{49}$ Das Oberste Volksgericht hatte zwar im Jahr 2001 auf eine Anfrage des Oberen Gerichts der Provinz Shandong in der Rechtssache Qi Yuling die unmittelbare Anwendung des Grundrechts auf Bildung ${ }^{50}$ erlaubt. Diese „Antwort“51 auf die Anfrage des Oberen Gerichts wurde allerdings im Jahr 2008 wieder aufgehoben, womit das Kapitel der unmittelbaren Anwendung von Verfassungsnormen vorerst zu einem Ende gekommen ist. ${ }^{52}$ Auch wenn die Verfassung in der Rechtsprechungspraxis keine Wirkung entfaltet, so ist die Anwendung und Auslegung der Verfassung im akademischen Diskurs allgegenwärtig.

\section{Verstoß gegen das Prinzip der sozialistischen Marktwirtschaft}

Es wird vorgebracht, die Erwerbsbeschränkung für Immobilien verstoße gegen die in der Verfassung verankerten Prinzipien der sozialistischen Marktwirtschaft. Art. 15 der Verfassung sieht in Abs. 1 und 2 vor, dass der Staat eine sozialistische Marktwirtschaft durchführt, die Wirtschaftsgesetzgebung verstärkt und die Makrosteuerung verbessert. Diese Vorschrift erfährt in der Literatur eine liberale, wenn auch praxisferne Auslegung, wonach der Staat sich auf die Vorgabe des Rahmens des Wirtschaftens zu beschränken habe. Zwar habe der Staatsrat nach Art. 89 Ziff. 6 die Kompetenz zur Leitung und Verwaltung der wirtschaftlichen Tätigkeit und der städtischen und ländlichen Entwicklung, doch ermächtige diese Vorschrift den Staatsrat nicht, in einzelne Rechtsgeschäfte der Wirtschaftssubjekte einzugreifen. ${ }^{53}$

$47 \mathrm{Zu}$ justiziellen Auslegungen in China vgl. Björn Ahl, Die Justizauslegung durch das Oberste Volksgericht der VR China - Eine Analyse der neuen Bestimmungen des Jahres 2007, Zeitschrift für Chinesisches Recht (2007), S. 251-258.

48 In den $\S \S 3$ bis 5 der Zitiervorgaben wird die Verfassung nicht als eine Rechtsquelle angeführt, die als Entscheidungsgrundlage herangezogen werden kann. Zuigao renmin fayuan guanyu caipan wenshu yinyong falü, fagui deng guifanxing falü wenjian de guiding (Bestimmungen des Obersten Volksgerichts über das Zitieren von normierenden Rechtsschriftstücken wie Gesetze und Rechtsnormen in Entscheidungsurkunden) vom 26.10.2009.

49 Tong Zhiwei, Xianfa shiyong ying zunxing xianfa benshen guiding de lujing (Die Anwendung der Verfassung soll dem ursprünglich in der Verfassung bestimmtem Weg folgen), Zhongguo faxue (2008) Nr. 6, S. 22-48.

50 Art 46 Zhonghua renmin gongheguo xianfa (Verfassung der VR China) vom 4.12.1982.

51 Pifu.

52 Tong Zhiwei, A Comment on the Rise and Fall of the Supreme People's Court's Reply to Qi Yuling's Case, Suffolk University Law Review, Vol. 43 (2010), S. 101-111, 109.

53 Tai Qianhong, Fangdichan shichang xingzheng guizhi yu zhengfu quanli bianjie (Administrative Regulierung des Immobilienmarktes und die Grenzen der Macht der Exekutive), Zhongguo faxue (2011) Nr. 4, S. 30-34, 31. 


\section{Verletzung des Privatvermögens und des Subsistenzrechts}

In der Erwerbsbeschränkung wird auch eine Verletzung des Rechts auf Privatvermögen gesehen. ${ }^{54}$ Der Erwerb von Immobilien gehöre zu den elementaren Rechtsgeschäften von Wirtschaftssubjekten und falle als Teil der Vertragsfreiheit unter den Schutzbereich des Art. 13 der Verfassung. ${ }^{55}$ In der verfassungsrechtlichen Argumentation stützt man sich auch auf den 2004 neu eingeführten Art 33 Abs. 3, wonach der Staat die Menschenrechte respektiert und schützt. Zu den grundlegenden Menschenrechten gehöre das Subsistenzrecht und zum Recht auf Subsistenz und einem würdevollen Dasein gehöre auch - soweit die wirtschaftlichen Verhältnisse es erlauben - der Erwerb einer Eigentumswohnung.

\section{Verletzung des Gleichheitssatzes}

Die Erwerbsbeschränkung differenziert zwischen Haushalten, die dauerhaft in Peking registriert sind und solchen, bei denen die Registrierung nur vorübergehend ist. Die letztere Gruppe wird gegenüber der ersteren schlechter behandelt, da sie auf den Erwerb von nur einer Immobilie beschränkt wird und der Erwerb an den Nachweis der Zahlung von Steuern oder Sozialversicherungsbeiträgen geknüpft ist. In der Literatur wird diese Schlechterstellung von nach Peking Zugezogenen als Verstoß gegen den Gleichheitssatz in Art. 33 Abs. 2 der Verfassung angesehen. ${ }^{56}$ Eine Rechtfertigung der Ungleichbehandlung könne sich auch nicht daraus ergeben, dass der stetige Zustrom von Auswärtigen für die Ressourcenknappheit in Peking verantwortlich ist und die Lebenshaltungskosten für die Einwohner Pekings drastisch erhöht hat. ${ }^{57}$

Die Diskriminierung zwischen Personen mit ländlicher und städtischer Haushaltsregistrierung besteht auch in anderen Bereichen und wird beispielsweise besonders deutlich, wenn an Hinterbliebene von tödlich verunglückten Personen aufgrund deren unterschiedlicher Haushaltsregistrierung unterschiedlich hohe Entschädigungszahlungen geleistet werden. Grundlage für die unterschiedlichen Standards bei der Berechnung der Entschädigung ist $\S 29$ der Auslegung des Obersten Volksgerichts zum Schadensersatz für Körperschäden aus dem Jahr 2003. ${ }^{58}$ Danach wird eine pauschalierte Entschädigung aufgrund von Durchschnittseinkommen vorgesehen, die nach Land- und Stadtbewohnern unterscheidet. Dies wird in der

54 Art 13 Abs. 1 lautet: „Das rechtmäßige Privatvermögen der Bürger ist unverletzlich.“ Abs. 2: „Der Staat schützt gemäß den gesetzlichen Bestimmungen das Recht der Bürger auf Privatvermögen und Erbschaft.“ Abs. 3: „Der Staat kann, wenn es das öffentliche Interesse erfordert, gegen Entschädigung privates Vermögen belasten oder enteignen.".

55 Tai, Fn. 53, S. 31.

56 Tian Feilong, „Xiangouling“ de falixue fenxi (Rechtstheoretische Untersuchung der Erwerbsbeschränkung), Kexue zhinang (März 2011), S. 32-35.

57 Tai, Fn. 53, S. 33.

58 Zuigao renmin fayuan guanyu shenli renshen sunhai peichang anjian shiyong falü ruogan wenti de jieshi (Auslegung des Obersten Volksgerichts zu einigen Fragen der Anwendung des Rechts bei der Behandlung von Fällen des Ersatzes von Körperschäden) vom 26.12.2003. 
Literatur unter dem Stichwort „gleiches Leben mit ungleichem Wert“ (tongming butongjia) scharf kritisiert. ${ }^{59}$ Es gibt schon seit einigen Jahren Bemühungen, das System der Haushaltsregistrierung grundlegend zu reformieren. ${ }^{60}$ Vor allem vor dem Hintergrund der Reformen, die in den beiden Pilotstädten Chongqing und Chengdu durchgeführt werden, erscheint die Anknüpfung an die Haushaltsregistrierung für den Wohnungskauf als ein großer Rückschritt.

\section{Verhältnismäßigkeit}

Ein weiterer Punkt der Rechtmäßigkeitsprüfung der Erwerbsbeschränkung ist die Frage der Verhältnismäßigkeit. In der Literatur wird weitgehend der Ausprägung des Verhältnismäßigkeitsprinzips gefolgt, wie sie im deutschen Recht mit den Prüfungsschritten Geeignetheit, Erforderlichkeit und Angemessenheit der Regelung vorgenommen wird. ${ }^{61}$ Das Verhältnismäßigkeitsprinzip findet sich auch in einem Rundschreiben des Staatsrats über die rechtmäßige Verwaltung. ${ }^{62}$ Darin heißt es, dass Verwaltungsakte nicht nur auf der Grundlage von Gesetzen, Verwaltungsrechtsbestimmungen und Verwaltungsvorschriften ergehen, sondern auch vernünftig und gerecht ${ }^{63}$ sein müssen. Ferner müssen die ergriffenen Maßnahmen geeignet und erforderlich sein. Wenn Behörden verschiedene Maßnahmen zur Verfügung stehen, um ein Verwaltungsziel zu erreichen, so muss dasjenige Mittel eingesetzt werden, welches die Rechte und Interessen der Betroffenen am wenigsten beeinträchtigt.

Von der Geeignetheit der Erwerbsbeschränkung ist auszugehen, wenn als Ziel der Maßnahme eine Reduzierung des Preisanstiegs für Wohnimmobilien in großen Städten angesehen wird. Die offizielle Statistik für die Immobilienpreise in den 70 größten Städten Chinas zeigt, dass die Preise für Wohnimmobilien nicht mehr stark ansteigen bzw. gleichbleiben oder sinken. Während im Januar 2011 die Immobilienpreise noch in 60 von 70 Städten anstiegen, sind im Dezember 2011 die Preise nur noch in 2 von 70 Städten gestiegen, in 52 Städten sind sie gefallen und in 16 unverändert geblieben. ${ }^{64}$ Die Frage der Kausalität des Erwerbsverbots für

59 Yang Tiaofang, Fansi pingdeng yuanze, hanwei shengming zunyan - guanyu tongming butong pan de sikao (Über den Gleichberechtigungsgrundsatz und die Verteidigung der Würde des Lebens Gedanken über „gleiches Leben mit ungleichem Wert“), Fazhi yu shehui (Dezember 2010), S. 273-274.

60 Kam Wing Chan and Will Buckingham, Is China Abolishing the Hukou System? The China Quarterly, Vol. 195 (2008), S. 586-606.

61 Vgl. zur Verhältnismäßigkeitsprüfung der Nutzungsbeschränkung von Kraftfahrzeugen im Stadtraum Peking: Jin Guokun, Bili yuanze shiye xia de jiaotong xianxing cuoshi (Maßnahmen der Verkehrsbeschränkung unter dem Blickwinkel des Verhältnismäßigkeitsprinzips), http://law.china.cn/ features/2008-12/31/content_2958187.htm.

62 Guowuyuan guanyu yinfa quanmian tuijin yifa xingzheng shishi gangyao de tonzhi (Mitteilung des Staatsrats über den Druck und die Verbreitung des Leitfadens für die Umsetzung der umfassenden Förderung der gesetzmäßigen Verwaltung) vom 22.3.2004.

63 Yao heli yao zhengdang.

64 Zhonghua renmin gongheguo 2011 nian guomin jingji he shehui fazhan tongji gongbao (Statistischer Bericht der VR China über die volkswirtschaftliche und gesellschaftliche Entwicklung im Jahr 2011) http://www.stats.gov.cn/tjgb/ndtjgb/qgndtjgb/t20120222_402786440.htm. 
den Preisrückgang lässt sich freilich nicht zweifelsfrei nachweisen. Für diese Entwicklung mögen auch andere Faktoren ausschlaggebend gewesen sein, besonders wenn man bedenkt, dass sich wirklich Kaufwillige nicht von den Erwerbsbeschränkungen abhalten lassen und Wege finden, die Regelung zu umgehen. ${ }^{65}$ Die Geeignetheit der Maßnahme lässt sich indes verneinen, wenn als Ziel der Erwerbsbeschränkung die Einschränkung von Immobilienspekulation im ganzen Land angesehen wird, da Investoren häufig auf kleinere Städte ausgewichen sind, in denen keine Erwerbsbeschränkungen gelten und Immobilienpreise bezogen auf ganz China auch 2011 noch angestiegen sind. ${ }^{66}$

Geht man davon aus, dass die Erwerbsbeschränkung geeignet ist, das Ziel der Reduzierung der Nachfrage und des langsameren Preisanstiegs im Immobiliensektor zu erreichen, so stellt sich daran anschließend die Frage, ob die Erwerbsbeschränkung auch erforderlich ist. Die in Rede stehende Erwerbsbeschränkung ist jedenfalls das mildere Mittel gegenüber einer völligen Unterbindung von Transaktionen im Immobilienmarkt. Man könnte an andere möglicherweise mildere Mittel wie die Einschränkung der Kreditvergabe für Immobilienkredite denken. Im Schrifttum wird häufig mit der pauschalen Verweisung auf mildere Mittel, die für die Steuerung von Immobilienpreisen zur Verfügung stünden, die Erforderlichkeit verneint. ${ }^{67}$ Auch die Angemessenheit im engeren Sinn wird verneint, da die Maßnahme mit solch einem erheblichen Rechtseingriff einhergehe. ${ }^{68}$

\section{Rechtsschutz gegen das Erwerbsverbot von Wohnimmobilien}

Im Rahmen des Rechtsschutzes gegen die hier besprochene Regelung wird zunächst untersucht, ob ein unmittelbares gerichtliches Vorgehen gegen das Rundschreiben der Pekinger Stadtregierung möglich ist. Daran anschließend wird der Rechtsschutz gegen Einzelmaßnahmen erörtert, die auf der Grundlage des Rundschreibens getroffen werden.

\section{Rechtsschutz unmittelbar gegen das Rundschreiben}

Die allgemeine Frage der Justiziabilität staatlicher Eingriffe in den Markt wird von nur wenigen Autoren erörtert. Für die Justiziabilität staatlicher Marktinterventionen wird ins Feld geführt, dass Handlungen des Staates in diesem Bereich mehr wirtschaftlicher als politischer

65 Etwa werden Fälle berichtet, in denen nicht in Peking dauerhaft registrierte Personen zum Wohnungskauf Scheinehen eingegangen sind oder Ehen zum Schein aufgelöst wurden, um weitere Wohnungen zu erwerben. Vgl. dazu Organisationskomitee, Fn. 20, S. 23.

66 Ning Qinghua, Lun fangwu „xiangouling“ yu xingzhengfa shang de bili yuanze (Über die Beschränkung des Immobilienerwerbs und das Verhältnismäßigkeitsprinzip im Verwaltungsrecht), Fazhi yu shehui (2012) Nr. 3, S. 268-269, 269.

67 Vgl. Fu Qilin/Wang Liang, Fn. 42, S. 67.

68 Gao Jun, Xiangouling de hefaxing tanxi - jianlun fangjia tiaokong zhong de fazhi wenti (Untersuchung der Rechtmäßigkeit des Erwerbsverbots und Diskussion des Problems der Rechtsstaatlichkeit der Steuerung von Immobilienpreisen), Shanxi shida xuebao shehui kexue ban, (2012), Nr. 2, S. $40-44,41$. 
Natur seien und gerichtliche Kontrolle zu einer sorgfältigeren Wirtschaftspolitik führen könne. Dagegen wird eingewandt, dass es sich traditionell um Handlungen im exekutiven Kernbereich handle, die wie Akte des Staates im außenpolitischen Bereich nicht überprüfbar seien. ${ }^{69}$ Eine verwaltungsrechtliche Klage unmittelbar gegen das Rundschreiben würde schon an $\S 12$ Ziff. 2 des Verwaltungsprozessgesetzes scheitern, wonach abstrakt-generelle verwaltungsbehördliche Regelungen nicht vor Gericht angefochten werden können. ${ }^{70}$

\section{Rechtsschutz gegen Einzelmaßnahmen und inzidente Prüfung des Rundschreibens}

Anders zu beurteilen ist der Rechtsschutz gegen eine behördliche Einzelentscheidung, etwa die Entscheidung der für die Eintragung des Immobilienerwerbs zuständigen Behörde. Die Behörde wird den Antrag auf Eintragung auf Grundlage des Rundschreibens ablehnen, wenn die dort aufgeführten Voraussetzungen des Erwerbsverbots vorliegen. Dann ist fraglich, ob das Rundschreiben inzident durch das Gericht überprüft werden kann, bevor dann auf der Grundlage des Rundschreibens die Rechtmäßigkeit der Einzelentscheidung beurteilt wird.

Als Prüfungsmaßstab für Einzelentscheidungen der Verwaltung sieht das Verwaltungsprozessgesetz Gesetze, Verwaltungsrechtsbestimmungen und lokale Rechtsbestimmungen vor. ${ }^{71}$ Verwaltungsvorschriften sind bei der Behandlung von Verwaltungssachen lediglich zu berücksichtigen bzw. heranzuziehen. ${ }^{72}$ Normative Dokumente werden im Verwaltungsprozessgesetz nicht genannt und ihre Behandlung im Verwaltungsprozess war daher bis zu einer Klärung durch das Oberste Volksgericht umstritten und ist bis heute problematisch. Sie werden nach überwiegender Auffassung nicht zu den formellen Rechtsquellen gezählt, da sie nicht im Gesetzgebungsgesetz erwähnt werden. ${ }^{73}$ Darauf beruft sich auch die Ansicht, die davon ausgeht, dass alle Einzelentscheidungen, die auf der Grundlage normativer Dokumente erlassen werden, mangels Rechtsgrundlage rechtswidrig sind. Gerichte könnten deshalb ohne Prüfung der Rechtmäßigkeit normativer Dokumente die Einzelentscheidung aufheben. ${ }^{74}$ Nach einer anderen Meinung können Verwaltungsakte auch auf normative Dokumente gestützt werden. Begründet wird dies mit der praktischen Erwägung, dass Verwaltungsbehörden

69 Vgl. etwa Hu Guangzhi, Lun hongguan tiaokong xingwei de kesuxing (Über die Justiziabilität von makroökonomischer Kontrolle), Xiandai faxue (2008) Nr. 2, S. 62-69; Yan Yunqiu und Li Dawei, Hongguan tiaokong xingwei kesuxing fenxi (Analyse der Justiziabilität von makroökonomischer Kontrolle), Zhongguo sheshui kexueyuan yanjiu shengyuan xuebao (2005) Nr. 1, S. 48-51.

70 Zhonghua renmin gongheguo xingzheng susongfa (Verwaltungsprozessgesetz der Volksrepublik China) vom 4. April 1989, deutsche Übersetzung bei Heuser, Fn. 6, S. 244-260.

$71 \S 52$ Verwaltungsprozessgesetz.

$72 \S 53$ Verwaltungsprozessgesetz.

73 Yao Ruimin, Guanyu guizhang he qita guifanxing wenjian zai hefaxing shencha zhong de diwei he zuoyong de sikao (Überlegungen zu Status und Funktion von Verwaltungsvorschriften und sonstigen normativen Dokumenten bei der Rechtmäßigkeitsprüfung), Xingzheng luntan (2008) Nr.6, S. 53-57, 56.

74 Luo Haocai, Zhongguo sifa shencha zhidu (Das chinesische System gerichtlicher Überprüfung), Beijing 1993, S. 295-96. 
in großem Umfang vom Erlass normativer Dokumente Gebrauch machten. Würden die Volksgerichte die Wirksamkeit dieser normativen Dokumente nicht anerkennen, würden sie damit einer großen Zahl von Verwaltungsakten die Rechtsgrundlage entziehen, was der wirtschaftlichen und gesellschaftlichen Entwicklung schaden würde. ${ }^{75}$

Nach einer vermittelnden Ansicht sind normative Dokumente ähnlich wie die im Verwaltungsprozess „,heranzuziehenden“ Verwaltungsvorschriften Gegenstand einer gerichtlichen Rechtmäßigkeitsprüfung. ${ }^{76}$ Dies wird damit begründet, dass die Wirksamkeit normativer Dokumente nicht voraussetzungslos anerkannt werden könnte, da es für sie weder eine strenge Regelung der Zuständigkeit und des Erlassverfahrens noch ein System der Registrierung und Überprüfung gebe. Diese Ansicht stützt sich maßgeblich auf eine Justizauslegung sowie einer Besprechungsnotiz des Obersten Volksgerichts, wonach nur rechtmäßige normative Dokumente als Rechtmäßigkeitsmaßstab für Verwaltungsakte herangezogen werden können. ${ }^{77}$

In $\S 62$ Abs. 2 dieser Justizinterpretation wird ausgeführt, dass Gerichte bei der Behandlung von Verwaltungssachen im Urteil gültige Verwaltungsvorschriften und sonstige normative Dokumente zitieren können. In der Besprechungsnotiz hat das Oberste Volksgericht klargestellt, dass es sich bei den sonstigen normativen Dokumenten nicht um eine formale Rechtsquelle handelt. Wenn die Gerichte die Rechtmäßigkeit eines Verwaltungsakts beurteilen, der auf der Grundlage eines sonstigen normativen Dokuments erlassen wurde, sind normative Dokumente nur rechtserheblich, wenn das Gericht ihre Rechtmäßigkeit festgestellt hat. Folglich können Gerichte bei der Überprüfung einer Einzelentscheidung, welche die Behörde auf ein normatives Dokument gestützt hat, inzident die Übereinstimmung des normativen Dokuments mit höherrangigem Recht prüfen. Eine Verwerfungskompetenz kommt den Gerichten aber nicht zu. Stellt das Gericht die Rechtswidrigkeit des normativen Dokuments fest, kann es dieses lediglich in dem zu entscheidenden Fall unberücksichtigt lassen. An sich bestünde folglich die Möglichkeit, das Erwerbsverbot der Stadt Peking auf seine RechtmäBigkeit hin zu prüfen. Eine inzidente Prüfung des normativen Dokuments ist auch im Widerspruchsverfahren nach $\S 7$ des Verwaltungswiderspruchsgesetzes möglich. ${ }^{78}$

75 Diese Ansicht wird referiert bei: Li Jie, Qita guifanxing wenjian zai sifa shencha zhong de diwei ji tiaoli tanxi (Diskussion und Analyse von Status und Wirkung sonstiger normativer Dokumente in der gerichtlichen Überprüfung), Xingzheng faxue yanjiu (2004) Nr. 4, S. 42-48, 43.

76 Ying Songnian, Xingzheng susong faxue (Verwaltungsprozessrecht), Beijing 1990, S. 290.

77 Zuigao renmin fayuan guanyu zhixing zhonghua renmin gongheguo xingzheng susongfa ruogan wenti de jieshi (Auslegung des Obersten Volksgerichts über einige Fragen der Durchführung des Verwaltungsprozessgesetzes der VR China) vom 24.11.1999; Zuigao renmin fayuan guanyu yinfa guanyu shenli xingzheng anjian shiyong falü guifan wenti de zuotanhui jiyao de tongzhi (Mitteilung des Obersten Volksgerichts über Druck und Verbreitung der Besprechungszusammenfassung über Fragen der Anwendung von Rechtsnormen bei der Behandlung von verwaltungsrechtlichen Fällen) vom 18.5.2004.

78 Zhonghua renmin gongheguo xingzheng fuyifa (Verwaltungswiderspruchsgesetz der VR China) vom 29.4.1999. 


\section{Praxis des gerichtlichen Rechtsschutzes}

Von der rechtsdogmatisch möglichen inzidenten Überprüfung des Rundschreibens ist im Kontext der gegenwärtigen Wirtschaftspolitik bislang noch kein Gebrauch gemacht worden, da Gerichte wirtschaftspolitisches Regierungs- und Verwaltungshandeln, das im Dienst des Wirtschaftswachstums und der gesellschaftlichen Stabilität steht, nicht in Frage stellen.

In der Praxis werden Gerichte Fälle, die sich unmittelbar gegen solche Maßnahmen wenden, nicht annehmen. Wenn Gerichte die Möglichkeit einer inzidenten Prüfung der Rundschreiben haben, werden sie deren Rechtmäßigkeit unterstellen. Die Gerichte hatten seit Anfang des Jahres 2011 bereits eine große Zahl zivilrechtlicher Fällen zu entscheiden, bei denen die Regelung der Beschränkung des Immobilienerwerbs als rechtmäßig unterstellt wurde. Dabei ging es vor allem um die Rückabwicklung von Immobilienkaufverträgen, wenn der potenzielle Erwerber der Immobilie vor der Vollendung des Erwerbstatbestands aufgrund der Erwerbsbeschränkung die Berechtigung zum Erwerb von Immobilien verloren hatte. ${ }^{79}$

Diese Rechtsprechungspraxis ist in China möglich, da Richter in ihren Entscheidungen nicht nur an das Recht gebunden sind, sondern auch an politische Vorgaben, die über verschiedene Wege auf die Entscheidung im Einzelfall Einfluss nehmen können. ${ }^{80}$ In der Beurteilung der Rechtmäßigkeit des Rundschreibens ist ausschlaggebend, dass hohe Immobilienpreise als potenzielle Ursache für soziale Unruhen angesehen werden und Richter unter der Doktrin der gesellschaftlichen Stabilität keinen Spielraum haben, der ihnen erlauben würde, von der Partei- und Regierungslinie des Erwerbsverbots für Immobilien abzuweichen.

\section{E. Vergleichbare Entwicklungen auf anderen Gebieten}

Vergleichbare staatliche Eingriffe finden sich auch bei der Zulassung und Nutzung von Kraftfahrzeugen in großen Städten wie Peking. Einerseits sieht die chinesische Regierung in der Automobilindustrie einen wichtigen Motor für die wirtschaftliche Entwicklung. Andererseits kann die Infrastruktur in den Stadtzentren nur eine begrenzte Zahl von neuen Fahrzeugen verkraften. Für die Stadt Peking gibt es Regelungen, die auf zwei Ebenen versuchen, diesen Widerspruch aufzulösen, einmal bei den Neuzulassungen und dann bei der Kraftfahrzeugnutzung im Stadtbereich. Die Neuzulassung von Kraftfahrzeugen in Peking wird seit Januar 2011 auf 240.000 pro Jahr beschränkt. Monatlich werden 20.000 Kennzeichen per Los vergeben. Ferner kann jeder Fahrer nur ein Kraftfahrzeug zulassen. Zur Zulassung berechtigt, sind Personen mit Pekinger Haushaltsregistrierung oder solche, die fünf Jahre in Folge Steuern oder Beiträge zur Sozialversicherung bezahlt haben sowie Ausländer, die sich seit mindestens einem Jahr in Peking aufhalten. Im Dezember 2010 waren 4,8 Mio. Kraftfahrzeuge in Peking

79 Organisationskomitee, Fn. 20, S. 22-24.

80 Beispielsweise durch vorgesetzte Richter, die Gerichtspräsidenten, den Rechtsprechungsausschuss, das übergeordnete Gericht, Regierungsorgane und Parteiorgane; vgl. zur Unabhängigkeit der Richter in China: Randall Peerenboom (Hrsg.), Judicial Independence in China: Lessons for Global Rule of Law Promotion, Cambridge 2010. 
zugelassen. ${ }^{81}$ Eine erste Regelung zur Beschränkung der Nutzung von Kraftfahrzeugen wurde im Zusammenhang mit den Olympischen Spielen im Jahr 2008 erlassen. Diese Maßnahmen dienten der Verbesserung der Verkehrssituation und der Luftqualität, um den störungsfreien Ablauf der Spiele zu gewährleisten. Dieses Modell wurde als so erfolgreich bewertet, dass nach Abschluss der Spiele eine erneute Verordnung der Pekinger Stadtregierung in Form einer so genannten Mitteilung erging, nach der die Nutzung von Kraftfahrzeugen tagsüber innerhalb der fünften Ringstraße von Montag bis Freitag jeweils an einem Tag entsprechend der Endziffer des Kennzeichens untersagt wurde. ${ }^{82}$ Diese Regelung wurde zwar in den Folgejahren etwas modifiziert, doch ist sie bis heute gültig. ${ }^{83}$ Aufgrund der derzeitigen Regelung wird an Werktagen die Geschwindigkeit des Verkehrsflusses durchschnittlich um 10\% erhöht und Abgasemissionen um 10\% gesenkt. ${ }^{84}$ Die Rechtslage ist derjenigen bei den Erwerbsbeschränkungen ähnlich: Auch in diesem Fall ist die Handlungsform der Verwaltung problematisch. Es gibt zwar im Verkehrsrecht und im Umweltrecht Rechtsgrundlagen, die zu Nutzungsbeschränkungen ermächtigen, doch sind danach nur zeitlich befristete Maßnahmen erlaubt. ${ }^{85}$ Als Kompensation der Nutzungsbeschränkung erhalten Fahrzeughalter einen Nachlass bei der Kraftfahrzeugsteuer.

\section{F. Eingriffe in den Immobilienmarkt und Rechtsentwicklung in China}

Abschließend werden die rechtswidrigen Eingriffe in den Immobilienmarkt unter den Gesichtspunkten des sozialistischen Rechtsstaats, des Normal- und Krisenmodus und der Abkehr vom Recht in den größeren Zusammenhang der Rechtsentwicklung in China eingeordnet.

\section{Sozialistischer Rechtsstaat}

Der Rückgriff auf ein weniger formalisiertes Instrument exekutiver Rechtsetzung, welches die formale Rechtsordnung für Maßnahmen der Eingriffsverwaltung an sich nicht mehr vor-

81 Chen Xin, Car License Plate Restrictions Cause Application Frenzy, China Daily vom 3.1.2011.

82 Nach dieser Regelung war das Fahren von Fahrzeugen mit Nummernschildern mit den Endziffern eins und sechs montags zwischen sechs und 21 Uhr untersagt. Beijingshi renmin zhengfu guanyu shishi jiaotong guanli cuoshi de tongzhi (Mitteilung der Volksregierung der Stadt Peking über die Ausführung von Verkehrsverwaltungsmaßnahmen) vom 27.9.2008.

83 Heute ist etwa das Fahren auf der fünften Ringstraße durchgehend für alle Fahrzeuge erlaubt, innerhalb des fünften Ringes gelten die Beschränkungen werktags jeweils von sieben bis 20 Uhr.

84 Xin Dingding, Beijing Car Restrictions to Continue, China Daily vom 6.4.2009.

85 Gao Kaishan, Weihao xianxing zhili Beijing jiaotong yongdu zhiyou duanqi xiaoying de boyi fenxi (Pekinger Management von Verkehrsstau durch Nutzungsbeschränkung aufgrund von Endziffern hat nur Kurzzeiteffekt - Analyse aus Sicht der Spieltheorie), Shangchang xiandaihua (2010) Nr. 5, S. 165-166; Jin Guokun, Fazhi zhengfu shijiao xia xingzheng juece de yaojian - Jiyu Beijingshi jiaotong xianxing cuoshi de kaoliang (Voraussetzungen von Verwaltungsentscheidungen nach rechtsstaatlichen Grundsätzen - Am Beispiel einer Untersuchung der verkehrsbeschränkenden Maßnahmen der Stadt Peking), Fazhi wenming (2009), Nr. 5, S. 50-53. 
sieht sowie der in der Praxis fehlende gerichtliche Rechtsschutz gegen solche Maßnahmen lässt sich mit dem Konzept des sozialistischen Rechtsstaats erklären. ${ }^{86}$ Mit der Annahme der Verfassung von 1982 durch den Nationalen Volkskongress hatte eine klare Abwendung von dem informellen System der Staatsleitung durch politische Kampagnen stattgefunden. Die Verfassung vollzog die formelle Trennung von Partei und Staat. Die einfache Gesetzgebung, vor allem das Verwaltungsprozessgesetz von $1989^{87}$ gaben dem Prinzip der Rechtsbindung der Verwaltung größeres Gewicht. Ein Schritt zur umfassenden Bindung der Macht von Partei und Staat an das Recht wurde damit aber nicht getan. Das Primat des Rechts im Sinne einer Autonomie des Rechts und einer Begrenzung und Kanalisierung des politischen Prozesses wurde bislang nicht verwirklicht und soll auch in absehbarer Zeit nicht verwirklicht werden.

Vielmehr gilt im „sozialistischen Staat der demokratischen Diktatur des Volkes“ ${ }^{\text {“88 }}$ das Primat der Politik und das Recht gilt lediglich im Rahmen der politischen Zweckmäßigkeit. Das Primat der Politik drückt sich in der Lehre von der führenden Rolle der Kommunistischen Partei und dem Instrumentalcharakter des Rechts aus. Ihre führende Rolle leitet die Partei aus ihrem Erkenntnismonopol ab, das sie aufgrund der Einsicht in die „objektiven Gesetze der gesellschaftlichen Entwicklung“ in Anspruch nimmt. ${ }^{89}$ Die Verfassung sieht keine Gewaltenteilung mit einer wechselseitigen Kontrolle der drei Staatsgewalten, sondern ein Volkskongresssystem vor, in dem alle Staatsorgane von den Volkskongressen geschaffen und beaufsichtigt werden. ${ }^{90}$ In der Verfassungspraxis sind die Organe der Exekutive von deutlich größerem Gewicht als die Volkskongresse.

Das Recht ist nur ein Instrument von mehreren, das der parteistaatlichen Führung zur Durchsetzung politischer Ziele zur Verfügung steht und welches nach politischen Zweckmäßigkeitsgesichtspunkten gesetzt und angewandt wird. Seit 1999 enthält die Verfassung die Verpflichtung zum Aufbau eines „sozialistischen Rechtsstaats. “91 Während der „rechtstaatliche Teil" des antithetischen Begriffspaars die Bindungswirkung des Rechts bezeichnet, geht es beim „sozialistischen Teil“ um die politische Zweckmäßigkeit der Rechtsanwendung, d.h. um Parteilichkeit und die Übereinstimmung mit den Erfordernissen der gesellschaftlichen Entwicklung. Welchem Element bei der Rechtsanwendung der Vorrang eingeräumt wird,

86 Dazu Björn Ahl, Ein Rechtsstaat chinesischen Typs? - Zur Verfassungsentwicklung in der VR China, Blätter für deutsche und internationale Politik (2006), S. 1380-1388; Björn Ahl, Beschlagnahme einer wissenschaftlichen Monographie durch das Flughafenzollamt Peking, Zeitschrift für Chinesisches Recht (2004), S. 42-45.

87 Vgl. Fn. 70.

88 Art. 1 Abs. 1 der Verfassung.

89 Die Verfassung von 1975 brachte den Grundsatz der Parteiführung deutlich darin zum Ausdruck, dass sie den Nationalen Volkskongress als Machtorgan unter der Führung der Kommunistischen Partei bezeichnete (Art. 16) und die Streitkräfte dem Vorsitzenden des Zentralkomitees der Partei unterstellte (Art. 15). In der geltenden Verfassung von 1982 wird auf die Führungsrolle der Partei nur in der Präambel eingegangen.

90 Art. 3 Abs. 3 der Verfassung.

91 Art. 5 Abs. 1 der Verfassung. 
bestimmt sich wiederum nach der aktuellen Parteipolitik. ${ }^{92}$ Wenn wie hier die gesellschaftliche Stabilität durch den rapiden Anstieg von Immobilienpreisen in Gefahr zu geraten scheint, tritt der Aspekt der Rechtsbindung in den Hintergrund und gegen das formale System verstoßende rechtswidrige Maßnahmen werden hingenommen.

\section{Normal- und Krisenmodus}

Die rechtswidrigen Eingriffe in den Immobilienmarkt können auch in Anlehnung an Heilmanns Unterscheidung eines Normalmodus und eines Krisenmodus der Entscheidungsfindung erklärt werden. ${ }^{93}$ Nach diesem Modell ist in Phasen der chinesischen Routinepolitik die Entscheidungsfindung durch lange Verfahren der Kompromissfindung gekennzeichnet. In Krisensituationen tritt ein Ausnahmemodus der Willensbildung in Kraft, der von Zentralisierung und Dekretierung politischer Entscheidungen geprägt ist. Merkmale des Krisenmodus sind Einengung der Spielräume der regionalen Regierungen durch innerparteiliche Weisungen, abrupte Zentralisierung des Entscheidungsprozesses und zentrale Durchgriffe über die Parteihierarchie, Verengung des Zeithorizonts, geringere Bereitschaft der Zentrale zu Konsultationen, Zusammenrücken der Elite sowie eine kurzzeitige Erhöhung der Durchsetzungskapazität. Der Krisenmodus tritt als Ad-hoc-Reaktion der zentralen politischen Führung auf Ereignisse auf, die als Bedrohung für die Stabilität des nationalen politischen Systems wahrgenommen werden. Rechtswidrige Eingriffe in den Immobilienmarkt als Reaktion auf den übermäßigen Anstieg von Immobilienpreisen passt gut in das Schema des Krisenmodus, bei dem rechtliche Maßstäbe, die im Normalmodus die Staatsorgane binden, zurücktreten.

\section{Abwendung vom Recht}

Ein weiterer Erklärungsansatz nimmt die Hypothese zum Ausgangspunkt, dass sich die chinesische Führung in der Folge des 17. Parteikongresses im Jahr 2007 allmählich von dem Kurs der Rechtsreformen abgewandt hat, dem sie seit den 1980er Jahren gefolgt ist. Diese Reformen waren geprägt von intensiver Rechtsetzungstätigkeit, verstärkter Rechtsbindung der Verwaltung, der Einführung von Rechtsschutzmechanismen, einer Professionalisierung der Gerichte und eines einheitlichen juristischen Staatsexamens. ${ }^{94}$ Auslöser der „Abkehr vom Recht" war die Überzeugung der politischen Führung, dass allein mit rechtlichen Mechanismen wenig gegen steigende Unzufriedenheit und Proteste der Bevölkerung und gegen eine

92 Zum vergleichbaren Begriff der ,sozialistischen Gesetzlichkeit“ Ferdinand Kopp, Das Verfassungsverständnis in den sozialistischen Staaten, in: Recht und Staat (1972), S. 573.

93 Heilmann, Fn. 8, S. 42-44.

94 Vgl. dazu Carl Minzner, China's Turn against the Law, American Journal of Comparative Law, Vol. 59 (2011), S. 935-984; Benjamin Liebman, A Return to Populist Legality? Historical Legacies and Legal Reform, in: Sebastian Heilmann / Elizabeth Perry (Hrsg.), Mao's Invisible Hand: The Political Foundations of Adaptive Governance in China, Cambridge 2011, S. 165-200; weniger kritisch etwa: Susan Trevaskes, Political Ideology, the Party, and Politicking: Justice System Reform in China, Modern China, Vol. 37 (2011), S. 315-344. 
Gefährdung der gesellschaftlichen Stabilität, d.h. des nationalen politischen Systems auszurichten ist. Die Reaktion auf zunehmende gesellschaftliche Konflikte und deren Unruhepotenzial ist eine stärkere politische Kontrolle der Gerichte, die mit einem Auftrag populistischer Rechtsanwendung Hand in Hand geht. ${ }^{95}$ Diese in den letzten Jahren vollzogene „Repolitisierung" der Justiz wird von kritischen Stimmen als eine Rückgängigmachung der in den letzten zehn Jahren durchgeführten Rechts- und Justizreformen bezeichnet. ${ }^{96}$

Auch wenn sich die rechtswidrigen Eingriffe in den Immobilienmarkt gut in das Erklärungsmuster einer Abwendung vom Recht einfügen, sollte die Akzentverschiebung, welche der Entwicklung eines Rechtssystems und einer funktionierenden Justiz momentan weniger Gewicht als früher beimisst, nicht überbewertet werden. Unter der neuen Führungsgeneration könnte ab 2013 der ursprüngliche Reformkurs weiterverfolgt werden. Jedenfalls können die hier besprochenen Maßnahmen auch mit dem Konzept des sozialistischen Rechtsstaats erklärt werden. Während die Praxis der Eingriffe sich auf den ersten Teil des Begriffspaars stützt, sind die rechtswissenschaftlichen Diskurse, welche die Praxis einhellig als rechtswidrig kritisieren, Ausdruck der Rechtsstaatlichkeit.

95 Vgl. Benjamin Liebman, A Return to Populist Legality? Historical Legacies and Legal Reform, in: Sebastian Heilmann / Elizabeth Perry (Hrsg.), Mao's Invisible Hand: The Political Foundations of Adaptive Governance in China, Cambridge 2011, S. 165-200.

96 Mo Shaping, Zhongguo lüshi mianlin de zhidu fengxian (Systemische Risiken, denen chinesische Anwälte ausgesetzt sind), in Caijing Wang vom 20.7.2010 http://www.caijing.com.cn/ 2010-07-20/110482410.html. Für eine weniger kritische Einschätzung siehe Susan Trevaskes, Political Ideology, the Party, and Politicking: Justice System Reform in China, Modern China 37 Nr. 3 (2011), S. 315-344. 